\title{
La réflexivité comme instrument de formation par l'alternance et de développement de l'activité professionnelle des enseignants
}

The development of professional activity by alternating classroom teaching and reflective analysis

\section{Stefano Bertone et Frédéric Saujat}

\section{OpenEdition}

Journals

Édition électronique

URL : http://journals.openedition.org/activites/790

DOI : 10.4000/activites.790

ISSN : $1765-2723$

Éditeur

ARPACT - Association Recherches et Pratiques sur les ACTivités

\section{Référence électronique}

Stefano Bertone et Frédéric Saujat, «La réflexivité comme instrument de formation par l'alternance et de développement de l'activité professionnelle des enseignants », Activités [En ligne], 10-2 I Octobre 2013, mis en ligne le 15 octobre 2013, consulté le 01 mai 2019. URL : http://journals.openedition.org/ activites/790; DOI : 10.4000/activites.790

\section{c) (i) (9)}

Activités est mis à disposition selon les termes de la licence Creative Commons Attribution - Pas d'Utilisation Commerciale - Pas de Modification 4.0 International. 


\title{
La réflexivité comme instrument de formation par l'alternance et de développement de l'activité professionnelle des enseignants
}

\author{
Stefano Bertone
}

ESPE de la Réunion, EA 4075, IRISSE, Allée des Aigues Marines / Bellepierre, 97487 Saint-Denis de la Réunion stefano.bertone@univ-reunion.fr

\section{Frédéric Saujat}

ESPE de l'Académie d'Aix-Marseille, EA 4671 ADEF, Aix-Marseille Université, Ifé-ENS Lyon, 32, rue Eugène Cas, 13248 Marseille Cedex 04 - frederic.saujat@univ-amu.fr

\begin{abstract}
The development of professional activity by alternating classroom teaching and reflective analysis. This study is part of a research program to provide on-going assessment of the reforms in teacher training currently underway in France. The focus of this particular study was on the training conditions which optimize preservice teachers' reflective analysis of their work. The results showed that the professional activity of preservice teachers develops as they restructure the meaning of their experience through two processes: (a) the mobilization of professional concepts learned during training interactions and (b) the affects that they experience in self-confrontation interviews which provoke a greater awareness of their concerns and a remobilization of the concepts learned during post-lesson interviews.
\end{abstract}

\section{KEY WORDS}

Teacher Training, Reflectivity, Development, Professional Activity, Self-Confrontation Interviews

\section{Introduction}

Cet article vise à présenter et illustrer un programme de recherche destiné à évaluer et accompagner la rénovation de la formation professionnelle initiale des enseignants en France. Initiée en 2002 (Encart du Bulletin Officiel du MEN n 15 du 11 avril 2002) et réactualisée en 2007 (Encart du $\mathrm{BO}$ du MEN n 9 du 1er mars 2007), cette rénovation institue le principe de l'alternance entre des temps de formation à l'Institut Universitaire de Formation des Maîtres (IUFM) et des temps d'enseignement en établissements scolaires. À l'heure où cette formation a quitté les IUFM et a été placée dans les universités, se pose de façon aigüe la question de l'utilité de l'alternance pour la professionnalisation des enseignants et plus en général des pratiques réflexives d'analyse et/ou de co-analyse de l'expérience de classe sur le développement de l'activité professionnelle des enseignants novices $(\mathrm{EN})$. En effet, le postulat qui a présidé à la réforme est celui d'une augmentation du niveau de connaissance disciplinaire et scientifique en amont de l'exercice du métier à plein temps, avec la suppression de l'année de professionnalisation alternant un demi-service d'enseignement en établissement du second degré et un mi-temps de formation en IUFM. Bien que la relation entre les analyses réflexives de pratiques et les formations théoriques en IUFM n'aient pas complètement donné satisfaction au regard des attentes de formation et de professionnalisation, l'alternance envisagée dans l'ancienne formule avait pour avantage de 
laisser un temps conséquent (mais coûteux) de formation en centre universitaire et diminuait la pression de l'exercice quotidien du métier ainsi que la charge de travail en classe. Dans le cadre de la création des Écoles Supérieures du Professorat et de l'Éducation (ESPE) en cours de conception, la place de l'alternance est donc entièrement à repenser pour éviter les dérives de la « première mastérisation » sans revenir pour autant à l'ancienne formule proposée dans les IUFM, qui n'avait pas donné satisfaction. Cet article a donc pour objectif de montrer qu'il est possible de poser un programme de recherche permettant de discuter le caractère réflexif des formations d'enseignants et d'apprécier comment les acteurs, EN, Conseillers Pédagogiques $(\mathrm{CP})$ et Formateurs Universitaires (FU), répondent actuellement à l'exigence institutionnelle de l'alternance en formation en vue d'un renouvellement des modalités de celle-ci. La visée principale de ce texte est de monter que : (Partie 1) des questions non triviales peuvent être posées sur la question de l'alternance et de son efficacité au travers d'une littérature internationale qui est particulièrement fournie, (Partie 2) qu'elles peuvent être documentées à partir de données recueillies sur la base d'une théorie de l'activité et (Partie 3) que les résultats obtenus permettent de penser autrement la place et le statut de la réflexivité dans le développement de l'activité professionnelle des enseignants novices engagés dans des dispositifs de formation par alternance.

\section{1.- Se former en alternance : l'efficacité discutée des pratiques réflexives dans le développement des préoccupations et du sens de l'activité}

En ce qui concerne l'utilité et l'impact des formations des EN fondées sur le conseil pédagogique et les pratiques réflexives (mentoring ou supervision), les résultats issus de la littérature scientifique internationale sont contrastés (Chaliès \& Durand, 2000). En effet, en même temps que la formation de terrain est considérée comme cruciale dans l'implication professionnelle des EN dans le métier (Freese, 2006; Scherff, 2008; Siebert, Clark, Kilbridge, \& Peterson, 2006) et dans la communauté de pratiques (Kelchtermans \& Ballet, 2002) en facilitant le passage d'une posture d'étudiant à une posture d'enseignant (Stanulis, Fallona, \& Pearson, 2002), elle apparaît également peu efficace dans leur formation, notamment en raison de l'attitude «protectrice» des tuteurs (Carver \& Katz, 2004). À cet égard, les entretiens de conseil pédagogique se limitent bien souvent à des suggestions indirectes et à des encouragements ayant peu d'impact sur le développement professionnel des EN (Orland-Barak \& Klein, 2005 ; Semeniuk \& Worrall, 2000). Tantôt centrés sur le soutien émotionnel, voire «compassionnel » (Darling-Hammond, 2001), au détriment de l'analyse des difficultés réelles rencontrées en classe par ces derniers (Abell, Dillon, Hopkins, McInery, \& O'Brien, 1995 ; Arredondo \& Rucinski, 1998 ; Clarke \& JarvisSelinger, 2005 ; Edwards, 1997 ; Kwan \& Lopez-Real, 2005), tantôt fondés sur des malentendus ou des consensus apparents sur la compréhension de la situation analysée (Borko \& Mayfield, 1995 ; Bullough, Draper, Smith, \& Birrell, 2004 ; Paris \& Gespass, 2001), ces entretiens sont moins des occasions d'échange et de construction personnelle d'un style d'enseignement par les EN (Yayli, 2008) que des circonstances où les conseillers pédagogiques (CP) livrent leurs propres modèles d'action (Franke \& Dalhgren, 1996; Parker-Katz \& Bay, 2008).

Les entretiens de conseil pédagogique sont considérés comme efficaces lorsqu'un partenariat réflexif et collaboratif est établi (Chaliès, Ria, Bertone, Trohel, \& Durand, 2004 ; Fairbanks, Freedman \& Kahn, 2000), permettant l'analyse conjointe des difficultés professionnelles des EN (Feiman-Nemser, 2001 ; Hasher, Cocard, \& Moser, 2004). Les CP aident ainsi les EN à construire un point de vue personnel sur les questions d'apprentissage ainsi que sur leur propre «teaching style » (Pajak, 2001), tout en tissant des liens avec les savoirs théoriques dispensés à l'Université (Ottesen, 2007).

En ce qui concerne les interactions de formation entre enseignants novices et les Formateurs 
Universitaires (FU), les interactions en pointillé (Borko \& Mayfield, 1995 ; McNally, Cope, Inglis, \& Stronach, 1997) et le caractère théorique des échanges (Blake, Hanley, Jennings et Lloyd, 1996 ; Christie, Conlon, Gemmell, \& Long, 2004) sont perçus comme responsables de l'inefficacité relative de la formation. L'inefficacité de ces situations de formation est principalement liée au caractère théorique abstrait des concepts abordés et des analyses opérées (Darling-Hammond, 2006). Cela accentue chez les enseignants novices l'impression de « choc de réalité » (Veeneman, 1984), à l'égard des savoirs académiques appris : ces savoirs apparaissent le plus souvent comme inexploitables dans l'exercice quotidien du métier. L'activité des FU est parfois considérée comme inefficace même lorsque ces derniers s'engagent dans l'échange de formation à côté des $\mathrm{CP}$, notamment en raison de la discordance des points de vue (Beck \& Kosnik, 2002; Fairbanks, et al., 2000), des différences de culture et de formation (Kortaghen, Loughran \& Lunenberg, 2005; Smith, 2005) et des tensions engendrées par la confusion des rôles et des statuts qui émerge au cours de l'échange de formation (Snow-Gerono, 2008; Wilson, 2006; Yayli, 2008). Plus rarement, dans le cadre de dispositifs non-ordinaires de formation, un impact réel de la supervision en triade sur le développement de l'activité professionnelle des EN a été observé, notamment lorsque les FU interrogent les EN de façon complémentaire au CP et s'intéressent davantage à leurs préoccupations, décisions et sentiments qu'à l'efficacité de leurs actions (Cartaut \& Bertone, 2009 ; Chaliès, Bertone, Flavier, \& Durand, 2008).

Le point commun de la quasi-totalité des dispositifs de formation, est qu'ils organisent l'apprentissage du métier enseignant à partir de pratiques réflexives censées développer chez les EN le pouvoir d'analyse de leur expérience d'enseignement (Bertone, Méard, Ria, Euzet \& Durand, 2003 ; Parsons \& Stephenson, 2005 ; Ward \& McCotter, 2004), la construction de connaissances professionnelles (Chaliès, et al., 2004 ; Meijer, Zanting, Verloop, 2002 ; Oosterheert \& Vermount, 2003 ; Ponte, Ax, Beijaard, \& Wubbels, 2004) et leur pouvoir d'action en classe (Bertone, et al., 2006 ; Clarke, 2006 ; Feiman-Nemser, 2001). Cette littérature apparaît toutefois comme hétérogène à l'égard des paradigmes théoriques et méthodologiques retenus. Corollairement, on constate la présence de résultats contrastés, et parfois même contradictoires, qui ne permettent pas de discuter en profondeur la validité du postulat selon lequel il est possible de transformer les conceptions et/ou les pratiques professionnelles en faisant du travail réalisé en classe un objet de pensée et d'interlocution en dehors de son contexte de réalisation. Qui plus est, rares sont les études qui s'attachent à documenter et/ou discuter ce postulat par la production de données empiriques, même si des résultats existent qui montrent la dynamique intrapsychique de l'activité décisionnelle et des préoccupations des EN en classe (Ria, Sève, Saury, Theureau, \& Durand, 2003), sous l'effet des interactions de formation (Bertone, et al., 2003) et au cours de ces dernières (Chaliès, et al., 2004). Or, ce point nous semble déterminant pour concevoir un programme de recherche destiné à apprécier l'efficacité des dispositifs de formation fondés sur l'alternance. Cet article démontre la pertinence d'un programme de recherche sur la formation des EN qui viserait à comprendre et formaliser les conditions auxquelles la co-analyse réflexive de leur expérience de classe peut engendrer (a) une meilleure connaissance de leurs préoccupations et difficultés d'enseignement et, en retour, (b) une ouverture de possibles nouveaux en rapport avec les concepts et actions professionnels co-construits.

La question spécifique à laquelle répond ce texte est la suivante : à quelles conditions une formation fondée sur la réflexivité des EN est pour eux une occasion de découvrir la nature de leur engagement en classe et de développer le sens de leur activité d'enseignement en envisageant de nouvelles possibilités d'action? 


\section{2.- Cadre théorique et méthodologique}

\section{1.- Les dimensions de l'activité en formation}

L'ergonomie de l'activité (Daniellou \& Rabardel, 2005) s'intéresse au travail de l'enseignant non seulement dans sa dimension productive (résultats du travail sur son objet), mais aussi dans sa dimension constructive (effets en retour de ce travail sur l'enseignant lui-même). De ce point de vue il est utile d'interroger la formation des EN sous l'angle de la prise en compte de ces deux dimensions. La question est de savoir sous quelles conditions les conseils prodigués et l'étayage apporté permettent d'articuler les préoccupations des EN au cours de leur activité (Leontiev, 1984 ; Rubinstein, 2007) en classe avec l'accroissement de leur efficacité professionnelle.

Nous postulons que la prise en compte de cette dimension constructive joue un rôle central dans la compréhension de l'activité de l'EN, qui se trouve aux prises avec de complexes négociations d'efficacité. Celles-ci sont issues de la confrontation entre la recherche d'une efficacité objective du travail réalisé auprès de ses élèves, d'une part, et la recherche d'une efficacité subjective dans la réalisation de ce travail, d'autre part (Saujat, 2007).

Les illustrations choisies dans cet article pour documenter le développement de l'activité des EN met tout particulièrement en évidence les tensions entre ces deux formes d'efficacité, dans la mesure où ces derniers sont soumis à une double contrainte : celle d'avoir à organiser l'apprentissage des élèves qui leur sont confiés, tout en s'occupant de leur propre apprentissage du métier dans lequel ils s'efforcent d'entrer. Même si l'on ne suit pas jusqu'au bout Wildman, Niles, Magliaro et McLaughlin (1989) pour voir dans cette situation des EN l'expression d'un «double métier » en classe, dans la mesure où il s'agit pour nous d'une seule et même activité au moyen de laquelle ils enseignent pour faire apprendre (dimension productive) et ils apprennent en enseignant (dimension constructive), il est clair que ce double apprentissage ne va pas sans tensions ni conflits. Les compromis (Roustan \& Saujat, 2008 ; Saujat, 2002) qu'ils mettent en œuvre pour tenter de gérer au mieux ces tensions au cœur de leur activité résultent des arbitrages qu'ils opèrent entre ce qu'on leur demande et ce que ça leur demande. Nous entendons par là tout ce qu'ils auraient voulu faire, ce qu'ils ne sont pas parvenus à faire, ce qu'ils se sont interdit de faire ou encore ce qu'ils se sont vus contraints de faire.

En d'autres termes il s'agit en formation de permettre aux EN de «faire quelque chose » de leurs préoccupations en les recyclant dans des «occupations » nouvelles avec leurs élèves : c'est le devenir de ces préoccupations dans leurs occupations qui ouvre ou ferme la voie au développement de leur activité.

Nous considérons, à partir d'une approche vygotskienne, qu'il faut concevoir ce développement à la fois sous l'angle de son autonomie et de sa dépendance, c'est-à-dire comme un processus évolutif interne contrôlé artificiellement de manière externe, comme un rapport dialectique entre interne et externe. En effet, ce processus «d'internalisationexternalisation» (Lawrence \& Valsiner, 2003) s'organise selon une temporalité et une logique qui lui sont propres, mais en fonction d'une direction qui est définie et fournie par l'étayage des formateurs (Berducci, 2004).

En ce sens l'activité de formation introduit des instruments psychologiques (Vygotski, 1985) dans des contextes artificiels d'analyse réflexive par l'EN de sa propre expérience. Ces instruments permettent aux EN de signifier autrement leur vécu et génèrent, ce faisant, des contradictions entre les significations liées aux formes de leur activité en classe et les significations nouvelles qui émergent à la faveur des interactions formatives. Le dépassement de ces contradictions développementales, pour prendre l'expression proposée par Holzkamp (1983), nécessite de leur part un processus ininterrompu d'automouvement (Vygotski, $1990 ; 1997)$ débouchant sur une réorganisation et une reconfiguration de leurs 
anciennes préoccupations.

\section{2.- Documenter et alimenter la réflexivité par la méthode de l'autoconfrontation}

Dans le cadre du programme de recherche développé dans cet article, la mise en œuvre de dispositifs de recueil de données est ordonnée à un double objectif : créer (a) les conditions d'un retour réflexif de la part des EN sur leurs propres expériences de classe et de formations et (b) un développement de ces expériences sous l'effet de cette réflexivité. L'autoconfrontation des EN à ces expériences constitue l'un des dispositifs retenus conciliant des visées transformatives et de production de connaissances sur l'activité réelle des EN en classe et en formation. Autrement dit, la mise en œuvre du cadre méthodologique de l'autoconfrontation ne revient pas à renier la pertinence de méthodes traditionnelles, en premier lieu l'observation directe, mais conduit à se situer délibérément du côté des méthodes dites « indirectes » (Clot, 2001), au sens où l'accès à l'expérience vécue se fait par la médiation d'une autre expérience. Nous cherchons par là à matérialiser l'une des leçons principales du travail de Vygotski : dans ce qu'il a de vivant, le vécu est à revivre. En effet pour lui la conscience n'est pas seulement représentation et organisation mentale d'une activité, mais rapport entre activités, expérience vécue d'expériences vécues (Vygotski, 1999), travail de liaison entre elles. "L'action passée au crible de la pensée se transforme en une autre action, qui est réfléchie », écrit Vygotski (1994, p. 226). C'est ce redoublement de l'action que nous cherchons à produire par le recours à l'autoconfrontation : l'activité initiale de l'EN en classe ou en formation est revécue et redécrite lorsqu'il est amené à la commenter, en visionnant le film dont elle a fait l'objet, en présence du chercheur. Ce nouveau contexte dialogique, qui mobilise les ressources d'interlocution et de conceptualisation nouvelles, ouvre un espace sémiotique où la conscience comme travail de liaison peut se déployer (Vygotski, 2003). L'EN peut alors s'engager dans un dialogue avec lui-même dans le cadre duquel il justifie, évalue, ses propres actes, s'en étonne éventuellement et en construit une vision nouvelle. Il s'agit donc véritablement d'ouvrir un champ de possibilités nouvelles à un entrecroisement d'activités elles-mêmes nouvelles, en l'occurrence dialogiques, dont le film du travail de l'EN, soit la part perceptible, observable, de son activité initiale, constitue la référence (Amigues, Faïta, \& Saujat, 2004 ; Clot \& Faïta, 2000 ; Saujat, 2009). L'originalité de l'autoconfrontation, en tant que méthode de sollicitation de l'expérience et des savoirs en actes, réside donc dans la libération des façons de signifier offertes aux sujets. Ainsi, les mises en relation possibles entre les énoncés et les situations et actions de référence peuvent devenir elles-mêmes objet de réflexion et de débat, à travers la reconnaissance de la pluralité des voix et des signes qui composent la dimension concrète des échanges verbaux. Le cadre méthodologique dans lequel s'inscrit l'autoconfrontation constitue de ce point de vue un « espace-temps » (Clot $\&$ Faïta, 2000) où se déploie un rapport dialogique au sens de Bakhtine $(1977,1984)$, c'est à dire irréductible à l'enchaînement des répliques entre deux sujets engagés dans une interaction. La motricité qui supporte et anime les dialogues ainsi initiés (Clot \& Faïta, 2000) ouvre la voie à un processus, qui est à la source même du développement de la pensée (Clot \& Leplat, 2005), au cours duquel les EN observés deviennent les observateurs et les interprètes de leur propre activité, en classe ou lors des interactions de formation. Dans ce cadre les chercheurs ont eu systématiquement recours à des procédures d'entretien lors desquelles les acteurs étaient confrontés à des traces audiovisuelles de leur propre activité. Le choix des entretiens d'autoconfrontation a été conforme à l'idée selon laquelle, en confrontant les EN à leur travail et en les mettant en situation d'être observés par autrui, on leur offre finalement un nouveau destinataire, le chercheur, pour leurs propres dialogues intérieurs à propos de cette expérience. Par ce biais on parvient à la fois à susciter un développement de la signification de l'expérience en question, en même temps qu'on parvient à mieux connaître le travail endogène de réorganisation de celle-ci par l'acteur (Clot \& Leplat, 2005). La procédure standardisée a consisté à suivre des binômes «formateur de terrain (conseiller pédagogique) - enseignant novice» au cours de l'année de titularisation (2 ${ }^{\text {ème }}$ année d'IUFM) et à 
enregistrer à plusieurs moments de cette année à la fois des leçons, des interactions de formation à propos de ces leçons et des entretiens d'autoconfrontation des acteurs à propos de leur activité lors des leçons ou des entretiens de formation. C'est de ce corpus de données que sont extraits les exemples qui suivent.

\section{3.- Penser autrement la place et le statut de la réflexivité en formation}

Les résultats présentés ici ont déjà été exploités dans le cadre de comptes rendus de recherche antérieurs. L'enjeu de cet article n'est pas de produire un compte rendu de recherche empirique de plus, mais d'illustrer le rôle potentiel de la réflexivité et de la coanalyse de l'expérience d'enseignement des EN dans le développement professionnel. Il s'agit de définir un objet d'intervention-recherche (Clot, 2008) en rapport avec un programme de recherche empirique sur la formation par alternance des enseignants.

\section{Illustration 1. L'analyse réflexive de l'expérience de formation et l'émergence de préoccupations nouvelles pour faire la classe}

L'illustration qui suit est composée d'une interaction de formation et de l'extrait de l'autoconfrontation de l'enseignante novice qui a suivi. Elle concerne un CP (T) et une EN (K) dont il avait la responsabilité. T et K partageaient dans leur emploi du temps une classe de $3^{\text {ème }}$ dans un collège du Sud de la France et avaient décidé de prendre en charge une leçon chacun, une semaine sur deux, l'autre étant présent pour observer et participer au bilan de la leçon menée et à la co-conception de la suivante. Le contexte du recueil des données était le suivant : K avait demandé de l'aide à ses formateurs à propos de la façon de contrôler et étayer l'activité de ses élèves, souvent distraits, voire indisciplinés. Conjointement, dans le cadre de son mémoire professionnel de deuxième année d'IUFM, $\mathrm{K}$ devait produire une analyse de ses difficultés professionnelles et évoquer des perspectives de développement. C'est dans cette visée qu'un chercheur et un formateur de l'IUFM ont négocié les conditions d'une collaboration et se sont déplacés pour recueillir des données utilisables à la fois comme support de son analyse par $\mathrm{K}$ et comme données pour le programme de recherche.

À l'issue d'une leçon de badminton en Éducation Physique et Sportive (EPS), T a considéré que l'appel des élèves en début de leçon n'avait pas été réalisé de façon satisfaisante et a interrogé $\mathrm{K}$ à ce propos.

\section{CP : L'appel pour toi c'est juste le côté administratif ou ça fait partie de la prise en main ? \\ EN : Heu... C'est la prise en main heu pour voir si... Bon déjà s'ils écoutent dès le début... Et ce qu'ils font là (indique des élèves éloignés du groupe sur l'écran)... Là ils me demandent de faire des matchs hein?}

L'objet de l'échange a concerné la nécessité de faire autrement l'appel pour prendre en main la classe. Le $\mathrm{CP}$ a étayé $\mathrm{K}$ en lui montrant qu'elle avait réduit l'appel à un moment de contrôle des présences alors qu'il aurait dû lui permettre de prendre en main la classe. La surprise de $\mathrm{K}$ et la construction d'un engagement nouveau, non suggéré par le $\mathrm{CP}$, à partir de l'observation de sa leçon à l'écran («pour voir si... Bon déjà s'ils écoutent dès le début... ») montrent la tentative de $\mathrm{K}$ de dépasser la contradiction, générée par l'étayage du $\mathrm{CP}$, entre sa préoccupation, qui était de noter les présents / absents et celle du $\mathrm{CP}$, qui était en rapport avec l'efficacité du contrôle de la classe.

L'auto-confrontation du CP éclaire quant à ses motifs.

Ch. : Là tu lui poses une question sur la prise en main, elle te fait une réponse et ...

CP : Euh... Parce que son regroupement de classe par rapport à sa maîtrise du groupe est un peu en décalage. Quelles que soient ses classes elle se contente de faire un regroupement spontané, elle ne gère pas trop les élèves qui sont à la périphérie du groupe. Pour l'instant je la laisse vivre. Si de son côté elle le vit bien, je n'ai pas à intervenir, car il n'y a pas de conséquences négatives. 
L'auto-confrontation de $\mathrm{K}$ informe quant au fait qu'elle ne s'attendait pas à être interrogée sur l'appel, et montre sa dépendance à l'égard du $\mathrm{CP}$ au cours de cette étape initiale de l'analyse réflexive de son expérience professionnelle.

Ch. : Là, il te dit : "l'appel c'est juste le côté administratif, etc.». Qu'est-ce que tu penses de cette remarque?

EN : Euh... En fait, oui, je ne m'attendais pas trop à cette question sur l'appel. Mais c'est vrai que c'est pertinent parce que... Bon peut-être que j'aurais dû euh... Plus m'occuper de ceux qui étaient derrière ou... Il y a beaucoup de choses qui sont induites après par l'appel... En fait, je n'avais pas trop d'attentes sur cette phase-là. Donc c'est vrai que... Ça ne m'interpelle pas trop, la phase de l'appel.

Les résultats montrent que $\mathrm{K}$ a reconnu l'importance du début de la leçon («c'est vrai que c'est pertinent») et qu'elle a construit un engagement nouveau au cours des entretiens de conseil pédagogique. Cet engagement a rendu possible la conception d'actions nouvelles à réaliser («j'aurais dû euh... Plus m'occuper de ceux qui étaient derrière »), mais principalement au cours de l'autoconfrontation relative à la formation de terrain et donc à l'insu de son CP. Malgré cela, lors d'une leçon de tchouk ball deux mois et demi plus tard avec la même classe, $\mathrm{K}$ a fait l'appel en permettant à nouveau à certains élèves de discuter entre eux, de jouer avec les ballons, tout en répondant plus ou moins distraitement à l'appel de leur nom. Le CP a questionné K à propos des conditions de déroulement de l'appel lors du deuxième entretien de conseil pédagogique qui a suivi la leçon.

CP : Est-ce que les conditions de l'appel te conviennent?

EN : Eux, bon, je les ai rappelés plusieurs fois, mais là ce que... Enfin l'appel, disons qu'ils n’ont pas besoin d'être super, super attentifs. Après là, je leur dis "OK, écoutez-moi », mais pendant l'appel même... Il y a les garçons d'un côté, les filles de l'autre.

L'objet de l'interaction de formation a concerné le fait que les conditions de l'appel étaient mauvaises pour prendre en main la classe. $\mathrm{K}$ a reconnu le manque d'attention des élèves, mais a été hésitante dans sa réponse et a justifié son action («Enfin l'appel, disons qu'ils n'ont pas besoin d'être super, super attentifs »). Alors que deux mois et demi plus tôt $\mathrm{K}$ avait reconnu l'importance de la remarque du CP, lors de ce dernier entretien elle a été en désaccord avec lui. À l'issue de cette interaction, on pourrait avancer que le désaccord observé est en rapport avec une conception différente de l'action de faire l'appel et qu'il a été à l'origine d'un étayage manqué. L'auto-confrontation de $\mathrm{K}$ montre au contraire l'émergence de préoccupations nouvelles chez elle.

EN : Là je suis en train de lui dire qu'ils n'ont pas besoin d'être super attentifs, mais je pense que c'est à cause de ça qu'après, la séance a continué comme ça. Parce que dès le départ ils n'ont pas senti qu'il fallait qu'ils écoutent. Et je n'ai pas attendu qu'il y ait le silence alors qu'à ce moment-là j'aurais dû attendre. Là quand je faisais l'appel c'était administratif pour noter les absents, c'est tout ce que ça voulait dire pour moi.

$\mathrm{K}$ a reconnu ses difficultés et en a identifié les conséquences sur l'attention des élèves au cours de la leçon («parce que dès le départ ils n'ont pas senti qu'il fallait qu'ils écoutent»). Le fait que $\mathrm{K}$ ait évoqué les arguments énoncés deux mois et demi plus tôt par le CP pour interpréter ses difficultés («Là quand je faisais l'appel c'était administratif pour noter les absents») montre un impact significatif de l'étayage du CP sur le développement de l'analyse réflexive de $\mathrm{K}$ à partir des instruments psychologiques construits en formation. La difficulté est que ce développement a été insoupçonné par le CP et qu'il a émergé (une fois encore) lors de l'autoconfrontation de $\mathrm{K}$ à propos de la formation de terrain.

C'est en effet au cours de cette interaction en milieu artificiel d'autoconfrontation que $\mathrm{K}$ a réussi à opérer un lien entre la situation de classe et celle de formation et à amorcer le dépassement des contradictions, qui avaient émergé plusieurs mois plus tôt, par la formalisation d'actions possibles nouvelles («je n'ai pas attendu qu'il y ait le silence alors 
qu'à ce moment-là j'aurais dû attendre »).

Ce constat, qui illustre 1'hypothèse théorique formulée dans cet article, permet également d'évaluer un dispositif de formation de terrain fondé sur la réflexivité. On peut notamment identifier les limites de l'étayage apporté, lorsqu'il n'organise pas suffisamment le rapport entre les préoccupations des EN et les conseils dispensés. On constate également que la mise en œuvre d'un dispositif artificiel d'autoconfrontation, faisant de l'expérience de formation un objet d'interlocution, a organisé la récupération et la réélaboration de l'expérience de classe selon les modalités présentées dans la Figure 1.

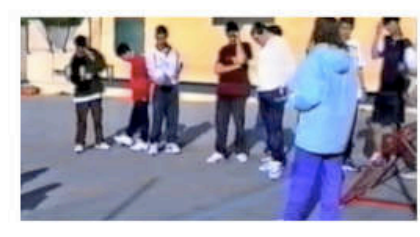

Leçon de

Tchouk Ball

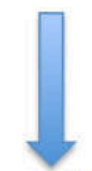

Formation de terrain

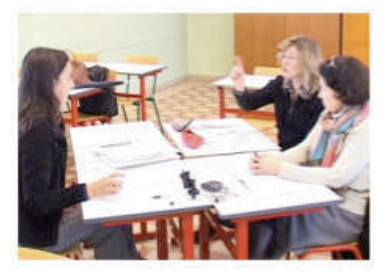

Autoconfrontation

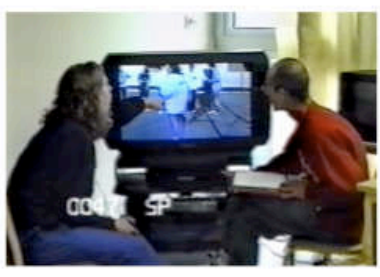

Figure 1 : Modélisation de la récupération de l'expérience de classe à partir de l'autoconfrontation relative à la formation de terrain

Figure 1: Model of the recovery of the class experience during selfconfrontation interviews on mentoring

Pour conclure cette analyse il convient de constater quelques limites des activités réflexives en termes de développement de l'activité de l'EN en classe et de poser une hypothèse de travail pour des recherches à venir. En effet, si dans le dispositif présenté les dimensions de normalisation et socialisations de l'activité ordinaire de formation de terrain semblent prendre le pas sur la fonction développementale du conseil pédagogique, l'autoconfrontation n'a pas permis non plus un développement probant de l'action en classe de l'EN. Ces données interrogent par conséquent les limites des co-analyses réflexives qu'elles s'inscrivent dans le contexte de la formation de terrain ou dans celui, moins habituel, des entretiens d'autoconfrontation. Si la valeur ajoutée de ces derniers apparaît en termes de réélaboration de l'expérience et de développement de l'activité perceptive et d'évaluation de son action en classe chez l'EN, en revanche on peut émettre l'hypothèse qu'il serait souhaitable que le suivi pédagogique du CP puisse s'étendre jusque dans les circonstances mêmes de la classe pour assister l'action de sa stagiaire et partager des pratiques efficaces in situ.

Illustration 2. L'analyse réflexive de l'expérience de classe et l'émergence de préoccupations nouvelles issues de la formation de terrain 
L'illustration qui suit est composée de l'extrait de l'autoconfrontation d'une enseignante novice (E) à une leçon de gymnastique en EPS conduite par elle avec des élèves de terminale d'un lycée du Sud de la France. Cette leçon avait fait l'objet d'un entretien à l'issue de son observation par une CP (M).

Ch. : L'impression qui persiste c'est qu'il y a quand même une réelle mise au travail, avec des régulations...

EN : Ouais, ouais... mais moi l'impression que j'ai en me voyant c'est que... en fait de saupoudrage comme me disait $M$, alors que j'avais pas l'impression de le faire pendant la séance, j'avais vraiment l'impression de m'intéresser à elles, et là quand je le vois j'ai vraiment... l'impression de... de passer et en fait de... à vouloir les regarder tous, je reste pas assez longtemps avec eux... je devrais peut-être prendre plus de temps avec un atelier à chaque fois.

Depuis le début de l'autoconfrontation E s'était contentée de réponses brèves et évasives lorsque le chercheur avait sollicité ses commentaires en arrêtant le déroulement du film, mais elle avait en revanche manifesté à plusieurs reprises des émotions ostensibles face au spectacle de son activité. Ici, en réponse à une intervention du chercheur qui lui a livré ses impressions, elle a, pour la première fois, évalué son activité en classe. La réappropriation de son activité au cours de l'autoconfrontation lui a permis de découvrir que sa préoccupation pendant la leçon était en fait relative au contrôle du groupe et à l'encadrement éducatif des élèves et non, comme elle en avait eu l'impression pendant la leçon, à la supervision des apprentissages aux différents ateliers.

Ch. : Est-ce que ce n'est pas le souci de...

EN : C'est le souci d'être avec tout le monde et voilà... mais là en les voyant je me dis : "est-ce que vraiment je les ai aidés? "Et je n'en suis pas si sûre que ça. Passer plus de temps... plutôt que de tourner. Parce que là mon principal objectif c'est de voir ceux qui ne travaillent pas vraiment et d'aller les bouger un peu... tout en essayant de regarder quand même ceux qui travaillent.

Ch. : Et c'est une remarque que t'avait faite M lors de l'entretien?

EN : M, oui ...

Ch. : Et lorsqu'elle te l'avait faite tu n'étais pas convaincue que...

EN : Non je n'étais pas convaincue du tout, j'avais vraiment l'impression d'être passée à chacune...

L'étonnement suscité par cette découverte a été d'autant plus marqué que la CP avait attiré son attention au cours de l'entretien sur les caractéristiques de son action de supervision pendant la leçon et que E avait été en désaccord avec l'interprétation proposée par la CP. La situation initiale, objet de la co-analyse, n'a pas été seulement « prétexte » à commentaire ou explication dirigés vers le chercheur, mais a constitué le contexte et l'objet d'une nouvelle activité nourrissant le dialogue ci-dessus. Ce contexte n'a donc pas seulement été ce à quoi se sont référés les commentaires de $\mathrm{E}$ au cours de l'autoconfrontation, mais aussi la source de ce qu'elle a fait et dit au moment où elle a redécouvert son travail à l'écran en mobilisant les ressources d'une interprétation pourtant jugée invalide au cours de l'entretien de formation. Son activité initiale et l'interprétation dont elle avait fait l'objet de la part de la $\mathrm{CP}$ ont pris alors sens d'une façon insoupçonnée, jusqu'alors, par E.

\footnotetext{
Ch. : Mais là ta préoccupation c'est vraiment de les mettre tous au travail...

EN : Ma préoccupation c'est ça, mais c'est vrai que là en me voyant je me dis que j'aurais été plus efficace en faisant heu...
}

La contradiction vécue entre ce qu'elle pensait faire et ce qu'elle s'est vue faire a alimenté 
un dialogue avec elle-même à partir du dialogue avec le chercheur, mais aussi avec la CP invisiblement présente, pour parler comme Vygotski, au cours de l'autoconfrontation. D'observée qu'elle a été initialement, dans des conditions où les conseils prodigués par la CP sont restés lettre morte durant l'entretien faute d'avoir été suffisamment articulés sur ses préoccupations effectives, E est devenue observatrice de son activité en autoconfrontation. Observatrice avec ses propres yeux bien sûr, mais également avec ceux de sa $\mathrm{CP}$. Ce contexte dialogique a permis alors, comme dans la première illustration, l'émergence de préoccupations nouvelles et leur recyclage possible dans des « occupations » plus efficaces (c'est vrai que là en me voyant je me dis que j'aurais été plus efficace en faisant heu...). Mais à la différence de l'illustration précédente, le développement initié par le dispositif d'autoconfrontation à l'expérience de classe a été ici étayé par la mobilisation de l'expérience de formation, récupérée et réélaborée selon les modalités présentées dans la Figure 2.
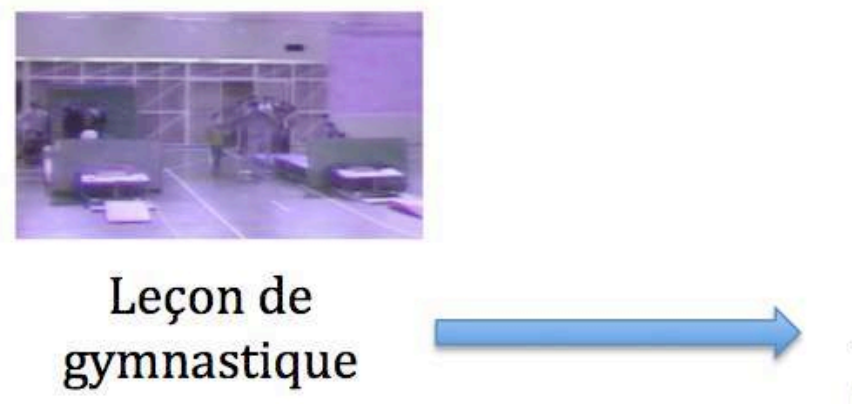

\section{Autoconfrontation}

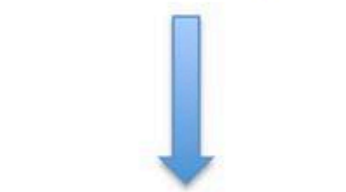

\section{Formation de} terrain
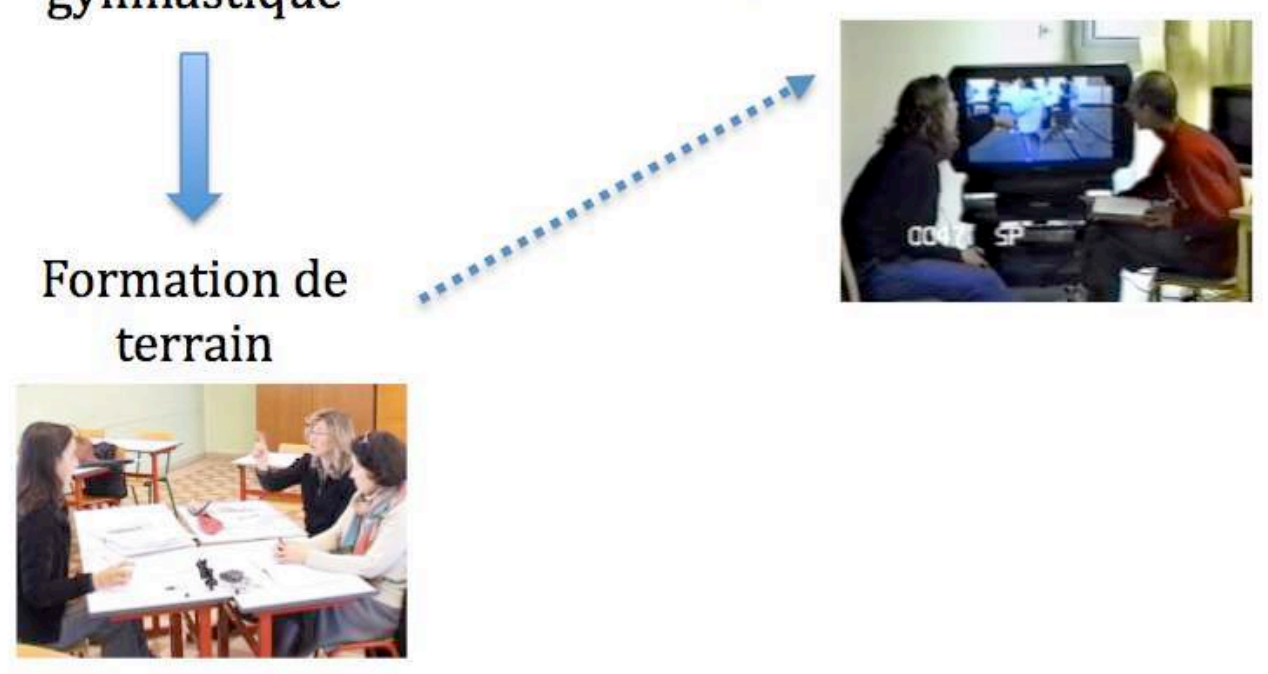

Figure 2 : Modélisation de la récupération de l'expérience de formation de terrain à partir de l'autoconfrontation relative à la classe

Figure 2: Model of the recovery of the mentoring experience during selfconfrontation interviews on the class

\section{Discussion}

Les extraits de résultats présentés ici montrent qu'il est possible de concevoir une formation qui se situe entre la découverte par les EN de leurs préoccupations effectives en classe et la co-construction de préoccupations nouvelles concurrentes. Ce processus de réorganisation du sens de l'expérience vécue s'accompagne d'un processus de resignification qui emprunte deux voies. La première voie de resignification apparaît comme le résultat de la mobilisation de concepts professionnels issus des interactions de formation sur l'expérience vécue par l'EN. Cette reconceptualisation de l'expérience, s'accompagne dans ce cas d'une réorganisation des préoccupations de l'EN, centrées non plus sur la tâche administrative de 
contrôle des présents, mais sur la fonctionnalité de l'appel comme instrument de prise en main de la classe. L'expérience émotionnelle de l'entretien de conseil pédagogique avec le refus (avoué en autoconfrontation par l'EN) de reconnaître la pertinence du conseil dispensé par le CP, a été motrice de la réorganisation des préoccupations de l'EN et d'une ouverture de possibles nouveau pour son action (là aussi énoncés en autoconfrontation).

La deuxième voie de resignification de l'expérience de la classe par l'EN s'est produite à l'occasion de l'expérience émotionnelle suscitée par l'entretien d'autoconfrontation. La découverte des préoccupations par $\mathrm{E}$ lors des interactions avec le chercheur a réactivé chez elle l'expérience de formation de terrain et lui a permis de remobiliser les concepts proposés par la CP au cours de l'entretien de conseil.

Dans une conception de l'apprentissage ancrée sur une théorie de l'activité collective (Engeström, 2001), le développement de possibles nouveaux peut être ici interprété comme le résultat d'une reconceptualisation de ses expériences quotidiennes par l'EN à partir des contradictions introduites par le collectif des formateurs et des chercheurs. Ces contradictions au regard d'une ancienne activité inscrite dans ses artefacts, règles et conventions ont été la source de transformations du sens de ces expériences, Engeström (1987).

Ces résultats permettent au passage de discuter des approches complémentaires de la formation de terrain (Six-Touchard \& Carlin, 2003) qui s'attachent principalement à montrer le rôle de la réflexivité, de l'auto-analyse, de l'abstraction et de la prise de conscience dans la construction de métaconnaissances et dans la formalisation des compétences nécessaires pour faire le travail. La réflexivité (dans la formation par alternance des apprentis cuisiniers), y est conçue par les auteurs comme un processus cognitif permettant de formuler les composantes de planification et contrôle de l'action ainsi que les opérations à réaliser pour transformer l'état initial de la situation en vue d'atteindre un état final, conformément à la modélisation de l'espace du problème en intelligence artificielle de Newell et Simon (1972). Nos résultats incitent plutôt à concevoir l'alternance en formation comme un espace de tensions émotionnelles entre la nécessité de s'appuyer sur les préoccupations actuelles des enseignants novices, et la nécessité de créer de la contradiction en vue de co-construire tant l'émergence de préoccupations nouvelles chez eux que les ressources opératoires permettant leur recyclage dans des occupations plus efficaces, pour eux comme pour leurs élèves. $\mathrm{Si}$, comme l'écrit Serres (2006), se former «c'est accepter de modifier avec prudence le début de stabilité professionnelle à peine échafaudée » (p. 207), alors la formation doit tenir le cap entre une stabilisation raisonnable de l'activité des enseignants novices lorsque celle-ci est vécue comme précaire ou critique, et une perturbation prudente lorsqu'elle a tendance à se figer dans des formes jugées insatisfaisantes du point de vue des protagonistes et/ou des formateurs (Ria, 2009). C'est dans cet espace de tensions que la formation peut entretenir le développement de l'activité, en s'efforçant de tenir à distance le risque de voir son fil se casser (Clot, 1995).

Le protocole mobilisé permet également de pointer le statut spécifique des artefacts de la formation dans le processus de resignification de l'expérience professionnelle. Si le premier cas étudié illustre l'impact des instruments psychologiques, le deuxième montre davantage l'effet des dispositifs artificiels d'analyse réflexive de l'expérience sur le développement professionnel. Mais les deux cas mettent en évidence les effets de la traversée de contextes différents dans l'analyse de leur activité par les EN. En les amenant à revivre une situation de classe ou de formation déjà vécues, l'autoconfrontation crée les conditions nécessaires pour que cette situation agisse sur eux afin qu'ils puissent en retour agir sur elle. Autrement dit, il semble nécessaire que les EN soient affectés par leur propre activité, en classe ou lors des interactions de formation, pour qu'ils soient en capacité d'élargir le champ des possibles subjectifs et opératoires de cette dernière.

La source de cet élargissement réside dans les alternances entre dialogue avec le chercheur, 
ou un formateur de centre dans le cadre d'un dispositif de formation en centre universitaire, et dialogue intérieur de l'EN avec lui-même à propos de son activité. Mais ses ressources sont les concepts et règles énoncés lors des interactions avec les différents acteurs de la formation, qui deviennent des instruments dont le sens «saute aux yeux» des EN dans ce nouveau contexte. Cela plaide en faveur de l'intérêt qu'il y aurait en formation à permettre aux EN de multiplier les points de vue sur leur propre expérience, en organisant une pluralité de contextes où ils peuvent entrer en « contact social avec eux-mêmes », pour reprendre une expression vygotskienne.

Il est également possible de défendre l'hypothèse selon laquelle ni le conseil pédagogique traditionnel, ni les artefacts introduits artificiellement dans la formation par la rechercheintervention, ne peuvent suffire à eux seuls à provoquer un développement réel de l'activité des EN.

L'enjeu principal du point de vue de la formation comme de la recherche-intervention est alors de s'y prendre «à plusieurs», «à plusieurs reprises» et dans «plusieurs circonstances », pour amener les EN à revivre des expériences vécues dont ils puissent envisager la transformation au moyen de leur interprétation avec les règles et les concepts professionnels de la formation, mobilisés, développés et réappropriés dans ces différents contextes (Bertone, Chaliès, \& Clot, 2009). Ce programme de recherche, en documentant spécifiquement la place et le statut de la réflexivité dans les différents moments de la formation par alternance, alimente la conception de dispositifs qui engagent des acteurs multiples dans des contextes dialogiques différents.

Il apparaît également que le dispositif de recherche introduit une analyse de l'activité de classe et de formation de terrain qui constitue un vecteur puissant d'évaluation itérative et de conception. À cet égard, par l'entrée «activité » qu'il propose et par la possibilité qu'il offre aux acteurs de la formation de (a) «ré-énacter» l'expérience de la classe et/ou de la formation par une sorte d'immersion mimétique dans le contexte de l'action et (b) de réélaborer l'expérience vécue dans le cadre d'une fiction (Durand, 2008) dialogique, ce protocole de recherche-intervention s'inscrit dans le cadre des approches de type technologique. C'est dans ce sens que ce texte plaide en faveur de programmes de rechercheintervention / recherche technologique au sein des futures ESPE, dont le travail d'évaluation et conception porte justement sur la mise en place de dispositifs de formation fondés sur l'alternance entre des apports théoriques exigés par la mastérisation et la professionnalisation requise pour exercer le métier dès la deuxième année de formation.

\section{BIBLIOGRAPHIE}

Abell, S.K., Dillon, D.R., Hopkins, C.J., McInerney, W.D., \& O’Brien, D.G. (1995). Somebody to count on: Mentor / intern relationship in a beginning teacher internship program. Teaching and Teacher Education, 11(2), 173-188.

Amigues, R., Faïta, D., \& Saujat, F. (2004). L'autoconfrontation croisée : une méthode pour analyser l'activité enseignante et susciter le développement de l'expérience professionnelle. Bulletin de psychologie, 469, 41-44.

Arredondo, D., \& Rucinski, T.T. (1998). Using structured interactions in conferences and journals to promote cognitive development among mentors and mentees. Journal of Curriculum and Supervision, 13(4), 300-327.

Bakhtine, M. (1977). Le marxisme et la philosophie du langage. Paris: Éditions de Minuit.

Bakhtine, M. (1984). Esthétique de la création verbale. Paris: Gallimard.

Beck, C., \& Kosnik, C. (2000). Associate teachers in pre-service education: Clarifying and enhancing their roles. Journal of Education for Teaching, 26(3), 208-224.

Berducci, D. (2004). Vygotsky through Wittgenstein: a new perspective on Vygotsky's developmental continuum. Theory \& Psychology, 14(3), 329-353.

Bertone, S., Chaliès, S., Clarke, A., \& Méard, J.A. (2006). The dynamics of interaction during post- 
lesson conferences and the development of professional activity: Study of a preservice physical education teacher and her cooperating teacher. Asia-Pacific Journal of Teacher Education, 34(2), 245-264.

Bertone, S., Chaliès, S., \& Clot, Y. (2009). Contribution d'une théorie de l'action à la conceptualisation et à l'évaluation des pratiques réflexives dans les dispositifs de formation initiale des enseignants. Le Travail Humain, 72(2), 104-125.

Bertone, S., Méard, J., Ria, L., Euzet, J-P., \& Durand, M. (2003). Intrapsychic conflict experienced by a preservice teacher during classroom interactions: A case study in physical education. Teaching and Teacher Education, 19, 113-125.

Blake, D., Hanley, V., Jennings, M., \& Lloyd, M. (1996). Change in teacher education: Interpreting and experiencing new professional roles. European Journal of Teacher Education, 19(1), 19-34.

Borko, H., \& Mayfield, V. (1995). The roles of the cooperating teacher and university supervisor in learning to teach. Teaching and Teacher Education, 11(5), 501-518.

Bullough, R.V., Draper, R.J., Smith, L., \& Birrell, J.R. (2004). Moving beyond collusion: Clinical faculty and university/public school partnership. Teaching and Teacher Education, 20, 505-521.

Carver, C.L., \& Katz, D.S. (2004). Teaching at the boundary of acceptable practice. What is new teacher mentor to do? Journal of Teacher Education, 55(5), 449-462.

Cartaut, S., \& Bertone, S. (2009). Co-analysis of work in the triadic supervision of preservice teachers based on neo-Vygotskian activity theory: case study from a French university institute of teacher training. Teacher and Teaching Education, 25(8), 1086-1094.

Chaliès, S., Bertone, S., Flavier, E., \& Durand, M. (2008). Effects of collaborative mentoring on the articulation of training and classroom situations: A case study in the French school system. Teaching and Teacher Education, 24, 550-563.

Chaliès, S., \& Durand, M. (2000). L'utilité discutée du tutorat en formation initiale des enseignants. Note de synthèse. Recherche et Formation, 35, 145-180.

Chaliès, S., Ria, L., Bertone, S., Trohel, J., \& Durand, M (2004). Interactions between preservice and cooperating teachers and knowledge construction during post-lesson interviews. Teaching and Teacher Education, 20(8), 765-781.

Christie, F., Conlon, T., Gemmell, T., \& Long, A. (2004). Effective partnership? Perceptions of PGCE student teacher supervision. European Journal of Teacher Education, 27(2), 109-123.

Clarke, A. (2006). The nature and the substance of cooperating teacher reflection. Teaching and Teacher Education, 22, 910-921.

Clarke, A., \& Jarvis-Selinger, S. (2005). What the teaching perspectives of cooperating teachers tell us about their advisory practices. Teacher and Teaching Education, 21, 65-78.

Clot, Y (1995). Le travail sans l'homme ? Paris: La Decouverte.

Clot, Y. (2001). Méthodologie en clinique de l'activité. L'exemple du sosie. In M. S. Delefosse et G. Rouan (Eds.). Les méthodes qualitatives en psychologie (pp. 125-147). Paris: Dunod.

Clot, Y. (2008). Travail et pouvoir d'agir. Paris: PUF.

Clot, Y., \& Faïta, D. (2000). Genre et style en analyse du travail. Travailler, 4, 7-42.

Clot, Y., \& Leplat, J. (2005). La méthode clinique en ergonomie et en psychologie du travail. Le Travail Humain, 68(4), 289-316.

Daniellou, F., \& Rabardel, P. (2005). Activity-oriented approaches to ergonomics: Some traditions and communities. Theoretical Issues in Ergonomics Science, 6(5), 353-357.

Darling-Hammond, L. (2001). When conceptions collide: Constructing a community of inquiry for teacher education in British Columbia. Journal of Education for Teaching, 27(1), 7-21.

Darling-Hammond, L. (2006). Constructing $21^{\text {st }}$-Century Teacher Education. Journal of Teacher Education, 57(3), 300-314.

Durand, M. (2008). Un programme de recherche technologique en formation des adultes. Une approche enactive de l'activité humaine et l'accompagnement de son apprentissage/développement. Éducation et Didactique, 2(2), 27-43.

Edwards, A. (1997). Guests bearing gifts: The position of student teachers in primary school classrooms. British Educational Research Journal, 23(1), 27-37. 
Engeström, Y. (1987) Learning by Expanding: an activity-theoretical approach to developmental Research. Helsinki: Orienta-Konsultit.

Fairbanks, C.M., Freedman, D., \& Kahn, C. (2000). The role of effective mentors in learning to teach. Journal of Teacher Education, 51(2), 102-112.

Feiman-Nemser, S. (2001). Helping novice learn to teach. Lessons from an exemplary support teacher. Journal of Teacher Education, 52(1), 17-30.

Franke, A., \& Dahlgren, L.O. (1996). Conceptions of mentoring: An empirical study of conceptions of mentoring during the school-based teacher education. Teaching and Teacher Education, 12(6), 627-641.

Freese, A.R. (2006). Reframing one's teaching: Discovering our teacher selves through reflection and inquiry. Teaching and Teacher Education, 22, 100-119.

Hascher, T., Cocard, Y., \& Moser, P. (204). Forget about theory-practice is all? Student teachers'learning in practicum. Teachers and Teaching: Theory and Practice, 10(6), 623-637.

Holzkamp, K. (1983). Grundlegung der Psychologie. Frankfurt: Campus.

Kelchtermans, G., \& Ballet, K. (2002). The micropolitics of teacher education. A narrativebiographical study on teacher socialisation. Teaching and Teacher Education, 18, 105-120.

Korthagen,F., Loughran, J., \& Lunenberg, M. (2005). Teaching teachers - studies into expertise of teacher educators: An introduction to this theme issue. Teaching and Teacher Education, 21, 107115.

Kwan, T., \& Lopez-Real, F. (2005). Mentors' perceptions of their roles in mentoring student teachers. Asia-Pacific Journal of Teacher Education, 33(3), 275-287.

Lawrence, J.A., \& Valsiner, J. (2003). Making personal sense: An account of basic internalization and externalization processes. Theory and Psychology, 13(6), 723-752.

Leontiev, A. (1984). Activity, consciousness, personality. Moscow: Progress Editions.

McNally, J., Cope, P., Inglis, B., \& Stronach, I. (1997). The student teacher in school: Conditions for development. Teaching and Teacher Education, 13(5), 485-498.

Meijer, P.C., Zanting, A., \& Verloop, N. (2002). How can student teachers elicit experienced teachers' practical knowledge ? Tools, suggestions and significance. Journal of Teacher Education, 53(5), 406-419.

Newell, A., \& Simon, H.A. (1972). Human problem solving. Englewood Cliffs, NJ: Prentice-Hall.

Oosterheert, I.E., \& Vermunt, J.D. (2003).Knowledge construction in learning to teach: the role of dynamic sources. Teachers and Teaching: Theory and Practice, 9(2), 157-173.

Orland-Barak, L., \& Klein, S. (2005). The expressed and the realized: Mentors' representations of a mentoring conversation and its realization in practice. Teaching and Teacher Education, 21, 379402.

Ottesen, E. (2007). Teachers "in the making": Building accounts of teaching. Teaching and Teacher Education, 23, 612-623.

Pajak, E. (2001). Clinical supervision in a Standards-Based Environment: Opportunities and challenges. Journal of Teacher Education, 52(3), 233-243.

Paris, C., \& Gespass, S. (2001). Examining the mismatch between learner-centered teaching and teacher-centered supervision. Journal of Teacher Education, 52(5), 398-412.

Parker-Katz, M., \& Bay, M. (2008). Conceptualizing mentor knowledge: Learning from the insiders. Teaching and Teacher Education, 24(5), 1259-1269.

Parsons, M., \& Stephenson, M. (2005). Developing reflective practice in student teachers: collaboration and critical partnership. Teachers and Teaching: Theory and Practice, 11(1), 5-116.

Ponte, P., Ax, J., Beijaard, D., \& Wubbels, T. (2004). Teachers' development of professional knowledge through action research and the facilitation of this by teacher educators. Teaching and Teacher Education, 20, 571-588.

Ria, L. (2009). De l'analyse de l'activité des enseignants en milieu difficile à la conception de dispositifs de formation. In M. Durand \& L. Filliettaz (Eds.). Travail et formation des adultes (pp. 217-243). Paris: PUF.

Ria, L., Sève, C., Saury, J., Theureau, J., \& Durand, M. (2003). Beginning teachers situated emotions: 
A study of first classroom experiences. Journal of Education for Teaching, 29(3), 219-233.

Roustan, C., \& Saujat, F. (2008). « Genre débutant » et co-construction d'un milieu pour l'étude : le cas du football au cours moyen (9-10 ans). Travail \& Formation en Education, 1/2008. Mis en ligne le 2 décembre 2008. URL : http://tfe.revues.org/index616.html.

Rubinstein, S. L. (2007). Nouvelles figures de l'activité humaine. Anthologie de textes choisis et édités par V. Nosulenko et P. Rabardel. Toulouse: Octarès-Editions, Maison des sciences de l'homme.

Saujat, F. (2002). Quand un professeur des écoles débutant instruit son «sosie » de son expérience. Les Dossiers des Sciences de l'Éducation, 7, 107-117.

Saujat, F. (2007). Enseigner : un travail. In V. Dupriez \& G. Chapelle (Eds.). Enseigner (pp. 179188). Paris: PUF.

Saujat, F. (2009). L'analyse du travail comme source et ressource de formation : le cas de l'orientation en collège. In M. Durand \& L. Filliettaz (Eds.). Travail et formation des adultes (pp. 245-274). Paris: PUF.

Scherff, L. (2008). Disavowed: The stories of two novice teachers. Teaching and Teacher Education, 24, 1317-1332.

Semeniuk, A., \& Worrall, A.M. (2000). Rereading the dominant narrative of mentoring. Curriculum Inquiry, 30(4), 405-428.

Serres, G. (2006). Analyse de la construction de l'expérience professionnelle au gré des diverses situations de formation initiale des enseignants du second degré. Thèse de doctorat en Sciences de l'Éducation non publiée. Université Blaise Pascal, Clermont-Ferrand, France.

Siebert, C.J., Clark, A., Kilbridge, A., \& Peterson, H. (2006). When preservice teachers struggle or fail: Mentor teachers' perspective. Education, 126(3), 409-422.

Six-Touchard, B., \& Carlin, N. (2003). Impacts de l'auto-analyse sur les opérateurs et leur travail. Congrès de la Société d'Ergonomie de Langue Française. XVIII Congrès de la SELF. Paris.

Smith, K. (2005). Teacher educators' expertise: What do novice teachers and teacher educators say? Teaching and Teacher Education, 21, 177-192.

Snow-Gerono, J.L. (2008). Locating supervision. A reflective framework for negotiating tensions within conceptual and procedural foci for teacher development. Teaching and Teacher Education, 24(6), 1502-1515.

Stanulis, R.N., Fallona, C.A., \& Pearson, C.A. (2002). Am I doing what I am supposed to be doing? Mentoring novice teachers through the uncertainties and challenges of their first year of teaching. Mentoring and Tutoring, 10(1), 72-81.

Veenman, S. (1984). Perceived Problems of Beginning Teachers. Review of Educational Research, 54(2), 143-178.

Vygotski, L.S. (1985). La méthode instrumentale en psychologie. In B. Schneuwly \& J.P. Bronckart (Eds), Vygotski aujourd'hui (pp. 39-48). Neuchâtel et Paris: Delachaux et Niestlé.

Vygotski, L.S. (1990). To the question of psychology et pedology. In F.A. Fradkin (Ed.), Research in pedagogics. Discussion of 1920s and the early 1930s (pp. 323-351). Moscow: Progress Publishers.

Vygotski, L. (1994).Défectologie et déficience mentale. Lausanne: Delachaux et Niestlé.

Vygotski, L. (1997). Pensée et langage. Paris: Éditions Sociales.

Vygotski, L. (1999). La signification historique de la crise en psychologie. Lausanne \& Paris: Delachaux et Niestlé.

Vygotski, L.S. (2003). Conscience, inconscient, émotions. Paris: La Dispute.

Ward, J.R., \& McCotter, S. (2004). Reflection as a visible outcome for preservice teachers. Teaching and Teacher Education, 20, 243-257.

Wildman, T., Niles, J., Magliaro, J., \& McLaughlin, R. (1989). Teaching and learning to teach: The two roles of beginnings teachers. Elementary School Journal, 89(4), 471-492.

Wilson, E.K. (2006). The impact of an alternative model of student teacher supervision: Views of the participants. Teaching and Teacher Education, 22(1), 22-31.

Yayli, D. (2008). Theory-practice dichotomy in inquiry: Meanings and preservice teacher-mentor teacher tension in Turkish literacy classrooms. Teaching and Teacher Education, 24(4), 889-900. 


\section{RESUME}

Cet article s'inscrit dans le cadre d'un programme de recherche destiné à évaluer la rénovation de la formation initiale des enseignants en France. Il étudie les conditions d'une formation fondée sur la réflexivité des Enseignants Novices (EN). Les résultats montrent que le développement de l'activité professionnelle des EN s'accompagne d'un processus de resignification de leur expérience suivant deux voies: (a) la mobilisation de concepts professionnels issus des interactions de formation; (b) les affects suscités, au cours d'entretiens d'autoconfrontation, ayant permis aux EN de découvrir leurs préoccupations et de remobiliser les concepts proposés par les tuteurs.

\section{MOTS-CLES}

formation des enseignants, réflexivité, développement, activité professionnelle, entretiens d'autoconfrontation

\section{REFERENCEMENT}

Bertone, S. \& Saujat, F. (2013). La réflexivité comme instrument de formation par l'alternance et de développement de l'activité professionnelle des enseignants. Activités, 10(2), 177-192, http://www .activites.org/v10n2/v10n2.pdf

Article soumis le 12 décembre 2012, accepté pour publication le 14/06/2013 